\title{
Special issue on child computer interaction
}

\author{
Edward Tse
}

Published online: 9 November 2013

(C) Springer-Verlag London 2013

Given the growth of child computer interaction research and of the rapid adoption of interactive technologies as teaching tools, next-generation HCI technologies play an important role in the future of education. Educators rely on technology to improve/adapt learning to the pedagogical needs of learners; thus, the HCI community needs to examine how these concepts can be matched to contemporary paradigms in educational pedagogy. The classroom is a challenging environment for evaluation; thus, new interaction techniques need to be established to prove the value of new HCI interactions in the educational space. This workshop provides a forum to discuss key HCI issues facing next-generation education ranging from whole class interactive whiteboards, small-group interactive multitouch tables, and individual personal response systems in the classroom.

Interactive whiteboards and other electronic learning tools and materials have experienced exponential growth across many countries in recent years. This has resulted in a growing acceptance of digital content for delivering lessons in the classroom. Interactive whiteboards have also been quite successful in education given the proliferation of lesson-relevant content easily accessed through the Internet. Building on the discussions of our last $\mathrm{CHI}$ workshop on the next generation of HCI and education, there is a growing need to understand how these emerging technologies (e.g., walls, tables, and mobiles devices in Fig. 1) will be leveraged in educational environments. As this next generation of HCI technology arrives in the classroom, educators are expecting researchers and

E. Tse $(\bowtie)$

SMART Technologies, 3636 Research Road N.W., Calgary,

AB T2L 1Y1, Canada

e-mail: edwardtse@ smarttech.com practitioners to determine how this technology will align with contemporary educational pedagogy.

New technologies such as multi-touch whiteboards and interactive tables (Fig. 1) can be used by teachers to aid communication and collaboration. Gestures serve as consequential communication [4] so a teacher scaling a calendar with two fingers is also directing attention to course materials. Instruction is very much linked to speech actions in real time, and modern speech processing is opening up a number of exciting opportunities. Technology can assist and hinder educational pedagogy. In this workshop, we take the approach that next-generation HCI technologies will have a significant role in learning if the technology is applied in a pedagogically appropriate way and rigorously evaluated in the field.

Below we begin the discussion with perspectives of educational pedagogy, cognitive and physical design challenges, multi-user content development, and a revisitation of evaluation in an educational setting.

\section{Educational pedagogy}

The paradigm shift from teacher-centric to learner-centric pedagogy $[1,3,6]$ is being mirrored by teaching technologies that support the activities of a single teacher to those that support individual learners and small-group activities. Student-teacher and student-student negotiation is a key aspect of self-reflection [11]. Digital technologies have the potential to support self-reflection by enforcing agreement for global actions and encouraging negotiation when learners do not agree.

A recent study reported by Piper and Hollan [9] on the effect of a multi-touch table in a small-group collaborative learning setting for a neuroscience class at UCSD 

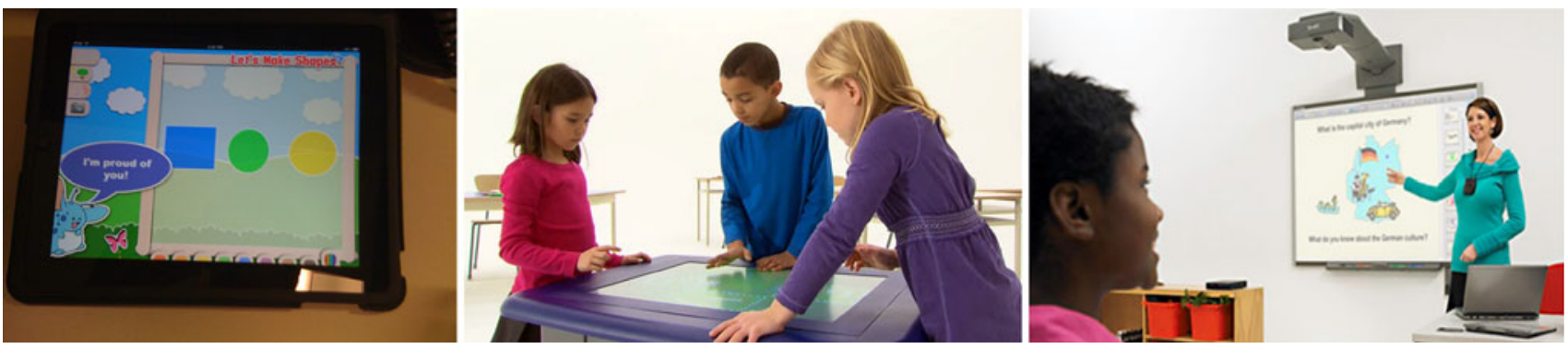

Fig. 1 Samples of next generation HCI technologies being applied to Educational Pedagogy. Shape drawing on the iPad (left), the multitouch SMART Table (middle) and an interactive whiteboard (right). SMART Technologies (middle, right)

demonstrated that the multi-touch table encourages students to experiment more with problem solutions. Computer systems are ideal for providing real-time feedback and validation to learners in a collaborative environment. This feedback can provide a sense of personal significance that is a part of constructivist learning $[5,10]$.

Educational pedagogy has long since recognized the value of active involvement in learning [1,7]. Technologies that respond to these actions such as mixed reality, direct manipulation, and multi-touch interfaces are only beginning to be applied to schools, and much of their potential to advance educational pedagogy requires further exploration by the research community.

\section{Design challenges}

When developing interfaces for children in the educational domain, many design challenges arise.

- Design for children

- Educational technology in the cloud

- Integrating mobile technology

\subsection{Design for children}

It is well know that children's cognitive and social skills develop over time [2,8], next-generation HCI techniques will need to consider both the physical and cognitive challenges that face young learners.

New HCI techniques will need to revisit the developmental cognitive skills of children especially in the 4-12 year age ranges. Cognitive skills such as memory load must be appropriate for the age level. Similarly, response time varies with younger age groups; thus, appropriate interaction times are important. Active involvement with the learning material is best achieved when the user interface is intuitive and unobtrusive, ensuring that children's concentration is maintained throughout the activity. Developing literacy skills also need to be considered in interface design where icons are used instead of text. Physical motor skills are a design factor when next-generation $\mathrm{HCI}$ devices are to be used by children. For example, fine motor skills are not fully developed yet in younger children; thus, precise object positioning can be a challenge. Interface designers need to consider appropriate target selection mechanisms, employing large widgets, and easy to perform drag and drop operations.

\subsection{Educational technology in the cloud}

Educational publishers and content providers are all actively looking at electronic delivery of their educational materials over the Web. For example, publishers are packaging some of their content in the ePub file format. The simplicity of downloading content has put educational content consumption into the hands of the individual learner. Creating educational content that captures and maintains the focus of learners remains a significant challenge. There is an increasing need for learners to collect information and share them as a group. Cloud-based technologies such as Google Documents can be used to support collaborative editing, but their implementations are not optimized for an educational setting. HCI design must consider a future where shared information resides in the cloud and that information can be accessed from any place in the world.

\subsection{Integrating mobile technology}

The use of mobile devices in the classroom has increased significant in recent years. This has placed new stresses on the network infrastructure of schools but has also changed the way that educational content is created and consumed. More importantly, online stores such as Apple's App Store make it possible for developers to financially benefit from creating content and applications are now being optimized for the mobile touch experience. As of this writing, the iPad alone has 3,000 educational applications available for purchase on the Apple App Store. Next-generation HCI devices have significant potential to capture real-time actions of participants and use this data for evaluation. There is a need to recognize the diverse types of learning 
environments (whole classroom, small groups, field trips, and informal learning) and determine metrics suitable for comparison. HCI work will need to synergize with efforts from the learning science and pedagogical communities to establish meaningful evaluation metrics for these new technologies and user interfaces.

\section{References}

1. Astin AW (1984) Student involvement: a developmental theory. Coll Stud Personnel 25:297-308

2. Bruckman A, Bandlow A (2003) Human-computer interaction for kids. In: Jacko J et al (eds) Handbook of human computer interaction. Lawrence Erlbaum Associates Inc., New Jersey, pp 428-440

3. Freire P (1970) Pedagogy of the oppressed. Herder and Herder, New York

4. Gutwin C, Greenberg S (1998) Effects of awareness support on groupware usability. In: Proceedings of the CHI 98, ACM Press, $511-518$
5. Bruner J (1996) The culture of education. Harvard University Press, Cambridge

6. Johnson D, Johnson R, Smith K (1998) Cooperative learning returns to college: what evidence is there that it works? Change 30(4):26-35

7. Montessori M (1912) The Montessori method. Frederick Stokes Co, New York

8. Piaget J (1972) Intellectual evolution from adolescence to adulthood. Hum Dev 15:1-12

9. Piper AM, Hollan JD (2009) Tabletop displays for small group study: affordances of paper and digital materials. In: Proceedings of the SIGCHI conference on human factors in computing systems (CHI '09). ACM, New York, pp 1227-1236. doi:10.1145/ 1518701.1518885

10. Rendon LI (1994) Validating culturally diverse students: toward a new model of learning and student development. Innov High Edu 19(1):33-51

11. Vygotsky LS (1978) Internalization of higher cognitive functions. Mind in society: the development of higher psychological processes. Harvard University Press, Cambridge. ISBN 0674576292 